\title{
La prise de conscience des représentations : première étape d'un projet de formation des enseignants
}

\section{Françoise Haramboure}

\section{(2) OpenEdition \\ Journals}

Édition électronique

URL : http://journals.openedition.org/asp/2844

DOI : 10.4000/asp.2844

ISSN : 2108-6354

Éditeur

Groupe d'étude et de recherche en anglais de spécialité

\section{Édition imprimée}

Date de publication : 1 décembre 1998

Pagination : 239-246

ISSN : 1246-8185

\section{Référence électronique}

Françoise Haramboure, «La prise de conscience des représentations : première étape d'un projet de formation des enseignants », ASp [En ligne], 19-22 | 1998, mis en ligne le 16 mars 2012, consulté le 19 avril 2019. URL : http://journals.openedition.org/asp/2844; DOI : 10.4000/asp.2844

Ce document a été généré automatiquement le 19 avril 2019

Tous droits réservés 


\title{
La prise de conscience des représentations : première étape d'un projet de formation des enseignants
}

\author{
Françoise Haramboure
}

1 Selon la définition d'Astolfi, les représentations recouvrent:

les conceptions qui dominent à chaque niveau d'âge et « le déjà-là conceptuel » que

l'enseignant a des chances de trouver, bien en place, quand il commence sa leçon... Quelque chose -quelque chose de structuré- préexiste bel et bien à la situation didactique et empêche le savoir du maître de se transmettre simplement aux élèves par un mécanisme d'émission-réception. (1993 : 27)

2 Pour les futurs enseignants d'anglais en formation initiale, ces conceptions en matière d'enseignement/apprentissage de la langue étrangère constituent un cadre de référence d'autant plus prégnant qu'il s'agit de sujets adultes pour lesquels l'apprentissage a représenté jusqu'ici l'essentiel des activités en termes de durée comme d'objectif. C'est donc au cours de leurs diverses expériences d'apprentissage qu'ils les ont progressivement construites. Elles dépendent non seulement de la réussite de ces expériences en terme cognitif mais aussi en terme affectif. Autrement dit, leurs conceptions sont liées à la fois à leurs performances dans cette discipline et aux sentiments d'estime de soi ou d'échec, de satisfaction ou d'insatisfaction qu'ils ont connus au cours de leur apprentissage. Elles sont également le reflet des attentes de la communauté culturelle à laquelle ils appartiennent. À ce titre, la priorité accordée depuis un certain nombre d'années au développement de la compétence de communication dans l'enseignement de l'anglais va certes de pair avec les travaux des chercheurs dans le domaine, mais elle reflète également l'influence grandissante de ce concept dans toutes les sphères de la vie sociale.

3 Il s'agit donc de conceptions complexes qui se sont stabilisées dans le temps et dont le poids a été décisif dans leur choix de la profession d'enseignant. Elles ont valeurde programme d'action, comme nous l'avons démontré précédemment s'agissant de l'effet des représentations des étudiants adultes sur leurs stratégies d'apprentissage de l'anglais. 
(Haramboure 1994 : 168-172). Elles ont un effet anticipateur et mobilisateur de l'activité des futurs enseignants: c'est à partir dece «déjà-là conceptuel » que les futurs enseignants abordent la formation pédagogique et didactique qui leur est proposée et qu'ils vont construire de nouveaux savoirs, savoir-faire et savoir-être.

4 En ce sens, l'exploration par les stagiaires de leurs représentations en matière d'apprentissage/enseignement de l'anglais s'inscrit dans une perspective constructiviste dans la mesure où elle met en jeu l'activité du sujet en formation et contribue à l'appropriation de nouveaux savoirs didactiques. Elle suppose l'élaboration d'outils d'investigation et la mise en oeuvre d'une démarche en cohérence avec le cadre théorique évoqué. Il importe d'en évaluer les effets sur le processus de formation pour en vérifier la pertinence.

\section{Présentation de l'outil}

Cette investigation peut recouvrir des procédures diverses et complémentaires, entretiens individuels, questionnaires fermés et questionnaires ouverts. Notre choix d'utiliser un questionnaire fermé ${ }^{1}$ a été motivé par la préoccupation de concilier nos responsabilités d'enseignant et de chercheur, c'est-à-dire de proposer une tâche pertinente, réalisable dans le cadre de la première séance de formation et qui pourrait nous fournir des données susceptibles d'étayer nos hypothèses de recherche. Ce faisant, si les informations obtenues sont moins approfondies, elles gardent néanmoins un caractère rigoureux et significatif. Par ailleurs, elles sont complétées par les réponses à des questionnaires ouverts, utilisés à mi-parcours de la première année dans le but de stimuler la réflexion métacognitive des stagiaires et les entretiens individuels effectués en cours d'année.

6 Le questionnaire regroupe vingt affirmations de caractère volontairement provocateur, dont le but est d'amener les étudiants à s'interroger et à réagir à l'égard de plusieurs domaines : leurs dispositions personnelles en matière d'enseignement, items 1 et 5 , les contenus et les démarches de formation, items $2,3,4,6$, la relation entre apprentissage et enseignement et par conséquent leur conception des rôles respectifs de l'enseignant et de l'élève, items $7,8,9,10,14,20$, la place relative qu'ils accordent aux savoirs et aux savoirfaire, items $11,12,13,15,16,17,18,19$. Par ailleurs, comme il est d'usage avec ce type d'outil, les réponses obtenues pour la plupart des items peuvent être croisées avec celles d'autres items pour vérification et/ou approfondissement. À titre d'illustration, la mise en relation des informations recueillies pour les numéros 9 et 10 permet une interprétation plus rigoureuse des conceptions des futurs enseignants en matière de traitement de l'erreur.

7 Cette liste est limitée dans le souci de ne pas disperser le questionnement des stagiaires au cours d'une activité qui leur est peu familière. Elle est nécessairement orientée par les représentations, les valeurs et la réflexion préalable de l'enseignant dans les domaines évoqués. Elle reflète ses priorités en termes de formation des enseignants d'anglais. À cet égard, le choix des rubriques 12 et 15 est révélateur de notre intérêt pour l'interaction langue/culture et de notre réflexion dans ce domaine. 


\section{La démarche}

8 Cette élucidation intervient au cours de la première séance afin que les jugements des stagiaires ne soient pas influencés par la réflexion sur l'enseignement/apprentissage de la langue étrangère qui est au centre de la formation.

9 La démarche proposée s'inscrit dans une perspective socio constructiviste. Comme le soulignent Williams et Burden, c'est dans les interactions en référence à un contexte particulier que les compétences individuelles se construisent :

four key sets of factors...influence the learning process : teachers, learners, tasks and contexts... Teachers select tasks which reflect their beliefs about teaching and learning. Learners interpret tasks in ways that are meaningful and personal to them as individuals. The task is therefore the interface between the teacher and learners. Teachers and learners also interact with each other... In addition to this, the context in which the learning takes place will play an important part in shaping what happens within it. (1997: 43-44)

10 La finalité de la tâche est double: la prise de conscience par les stagiaires de leurs représentations et de leurs implications, la construction du concept de représentation à travers cette expérience. Le langage et l'interaction entre pairs puis entre étudiants et enseignant ayant une fonction double, celle de faciliter le passage des représentations du niveau inconscient au niveau conscient et d'autre part de participer à la construction du concept.

11 Au cours d'une première étape, chacun des stagiaires sélectionne individuellement les affirmations et les classe en fonction de ses priorités. Il fait ensuite part de ses choix à un de ses partenaires afin de les expliciter et de les argumenter. Cette confrontation de points de vue exige de chacun des membres du binôme un travail de déconstruction et d'analyse qui l'amène à clarifier ses conceptions tant pour lui-même que pour son partenaire. Le rapport en séance plénière de la réflexion conduite en binôme se prolonge par une discussion des jugements retenus et de la hiérarchie établie, discussion orientée par l'enseignant sur leurs implications en matière d'enseignement/apprentissage.

\section{Discussion des résultats}

12 L'analyse des réponses fait apparaître une convergence surprenante dans le choix des items retenus. Tant pour les étudiants participant à un module de préprofessionnalisation au niveau de la licence que pour les stagiaires en première année d'IUFM, ce sont les items 4, 10,13, 15 qui retiennent en priorité leur attention. S'agissant de la hiérarchie établie parmi ces items, les items $n^{\circ} 13$ et 10 sont classés en première position regroupant respectivement $90 \%$ des réponses, les items $n^{\circ} 4$ et 15 en troisième position recueillant $60 \%$ des réponses. La quasi-unanimité que reflète ce classement souligne l'articulation étroite des niveaux individuel et collectif des représentations des étudiants.

13 En matière de contenus et objectifs de l'enseignement/apprentissage de l'anglais, la sélection des items 13 et 15 respectivement par $90 \%$ et $60 \%$ d'entre eux met clairement 
en évidence l'importance qu'ils attribuent au développement de la compétence de communication en anglais, recouvrant à la fois compréhension et expression en langue étrangère puisque moins de $1 \%$ des réponses privilégient l'expression seulement. Par ailleurs, en matière de contenus, ce choix manifeste clairement la priorité qu'ils accordent à l'acquisition des savoir-faire au détriment des savoirs, qu'il s'agisse de savoirs phonologiques, grammaticaux ou lexicaux qui sont invoqués dans moins de $1 \%$ des réponses.

Les étudiants se réfèrent, pour justifier ce choix, aux lacunes de leur propre expérience d'apprentissage orienté essentiellement vers l'acquisition de savoirs, notamment grammaticaux. Autrement dit, à travers ce jugement, ils expriment implicitement leur insatisfaction à l'égard des contenus et des objectifs de l'apprentissage de l'anglais et leur désir de changement. Ils se font l'écho de la demande sociale dans ce domaine. Seules les connaissances culturelles retiennent leur attention. Le plus souvent mises en relation au cours de la discussion avec la découverte de la communauté étrangère, elles sont perçues essentiellement comme une source parfois négligée de motivation et d'enrichissement pour les élèves. Leurs effets sur la communication en langue étrangère ne font pas l'objet de commentaires. De plus, la réflexion conduite en cours d'année montre leur perception réductrice de la culture étrangère, le plus souvent limitée à la description de comportements stéréotypés.

Leur insistance sur les savoir-faire est révélatrice d'une conception de l'apprentissage centrée sur le faire, c'est-à-dire sur l'engagement de l'élève. Cette interprétation est confirmée par la sélection de l'item $\mathrm{n}^{\circ} 10$ "L'erreur est une étape normale de l'apprentissage ». C'est le statut et le traitement de l'erreur qui suscitent les échanges les plus nombreux et les commentaires les plus négatifs. L'erreur est appréhendée par la majorité des étudiants comme le signe de l'activité de l'élève. Ils soulignent la nécessité d'un changement dans le domaine en évoquant les effets rédhibitoires d'une correction systématique des erreurs sur leur vécu de l'apprentissage de l'anglais tant en matière de performances que de motivation. Ils attribuent notamment les faiblesses des élèves en matière de communication en langue étrangère aux exigences excessives de correction grammaticale au détriment de la fluidité dans l'expression.

16 En terme de formation, leurs réponses font apparaître, à travers le choix de l'item 4, la nécessité de ne pas dissocier la réflexion théorique de la pratique de l'enseignement. Au cours de la discussion qui suit, ils soulignent implicitement le caractère formateur de l'apprentissage par l'expérience. Ils regrettent les limites de cette dimension en première année de formation initiale. Au demeurant, ils confirment ce point de vue dans les réponses fournies en cours d'année à un questionnaire ouvert dans lesquelles ils déplorent la brièveté des contacts avec les classes.

17 Par ailleurs, les items $1,5,7,14$ qui se référent aux dispositions spécifiques et à la fonction de l'enseignant d'anglais sont cités par une minorité d'entre eux. Ce refus de $97 \%$ des étudiants de se livrer à tout commentaire tant en matière de leurs dispositions personnelles pour l'enseignement que de certains aspects de la fonction enseignante, de la part de candidats à un concours de recrutement d'enseignants, suscite plusieurs interprétations. Il peut résulter de leur réticence à exprimer leur point de vue personnel sur leurs capacités personnelles dans ce domaine. Même s'ils évaluent spontanément les points forts ou les faiblesses de leurs enseignants, ils ne se livrent pas à ce même type d'analyse pour ce qui est de leurs propres dispositions pour l'enseignement. Comme les évocations nombreuses de leur expérience d'apprentissage au cours de la discussion 
tendent à le montrer, cette censure implicite semble révélatrice de leur perception de leur statut présent : ils se considèrent essentiellement comme des élèves/étudiants et s'interdisent en quelque sorte d'exprimer tout point de vue sur un domaine qui porte directement sur leur projet personnel. L'incertitude qu'ils éprouvent quant à sa réalisation n'est sans doute pas étrangère à cette attitude. Par ailleurs, cette absence de réaction à l'égard de ce qui relève du rôle de l'enseignant d'anglais témoigne également d'un manque de réflexion regrettable à la veille de leur engagement dans cette profession. Ce déficit peut être comblé par le travail de sensibilisation entrepris en amont dans les modules de pré-professionnalisation (Haramboure 1998).

\section{Les effets sur la formation}

Cette explicitation débouche sur une prise de conscience de conceptions familières demeurées inconscientes dans la plupart des cas jusqu'ici. À cet égard, les entretiens ponctuels conduits auprès des étudiants participant aux modules évoqués confirment notre analyse. Leurs hésitations et leurs difficultés à rendre compte des raisons qui les ont conduits à se spécialiser dans l'étude de la langue anglaise plutôt que dans celle de telle ou telle autre langue ou encore à verbaliser leurs conceptions de la culture angloaméricaine montrent qu'il s'agit de questions qui n'ont pas fait jusqu'ici l'objet de réflexion.

Cette prise de conscience contribue, notamment si elle s'accompagne d'une confrontation avec les points de vue de leurs partenaires, à une déconstruction et à une mise en perspective de ces points de vue, conditions sous-jacentes à l'appropriation de nouveaux savoirs. De ce fait, elle participe au déclenchement d'un questionnement, une remise en cause de certitudespropice à la construction des savoirs didactiques.

En l'absence de cette prise de conscience, la tension entre ces contenus et les conceptions des stagiaires est à l'origine des obstacles qu'ils rencontrent pour la construction de nouveaux concepts tels que ceux de "processus d'apprentissage» alors que traditionnellement ils envisagent l'apprentissage en termes de produits ou encore de résultats. Dans ce cas, l'on observe une superposition des concepts qui se traduit le plus souvent par des confusions au niveau terminologique. De ce fait se trouve également remise en cause l'élaboration de réseaux conceptuels, mettant en relation des concepts clés tels que "construction des savoirs ", "apprenant » et "conceptualisation » par exemple inhérents à l'appropriation de nouveaux modèles explicatifs concernant l'enseignement/apprentissage de l'anglais.

21 Parallèlement pour l'enseignant, cette explicitation constitue un diagnostic de départ. Elle lui permet d'identifier et d'anticiper les problèmes dus aux décalages que nous venons d'évoquer. Elle favorise le repérage des interférences entre les représentations des stagiaires et les informations nouvelles présentées. De ce fait, l'enseignant est en mesure de prévoir les situations de formation et les activités susceptibles de faciliter l'élaboration de nouveaux savoirs. À titre d'exemple, une démonstration théorique a priori est insuffisante pour permettre aux stagiaires de s'approprier le concept d'« interlangue ». Une confrontation avec des productions d'élèves est indispensable pour stimuler le questionnement, susciter l'explicitation d'hypothèses et tester l'opérationnalité et les limites de ce concept dans l'analyse de l'évolution des performances des élèves en anglais. La mise en relation de cette nouvelle donnée avec le modèle constructiviste contribue à son intégration dans un réseau conceptuel cohérent 
qui leur permet de renouveler leur lecture de la situation d'enseignement/apprentissage de la langue cible.

En revanche, si l'enseignant ne favorise pas cette phase d'investigation, il se prive également des points d'appui que constituent les représentations lors qu'elles sont en convergence avec les contenus de la formation. À cet égard, pour reprendre les résultats présentés, dès lors que pour les stagiaires, 'l'enseignement de la langue et de la culture ne peuvent être dissociés ", il importe que l'enseignant s'appuie sur ce jugement pour approfondir le concept de 'culture étrangère' et envisager les conditions du développement de la compétence culturelle dans le cadre de l'apprentissage guidé.

Enfin, à travers cette expérience, les futurs enseignants peuvent construire le concept de "représentation", et saisir l'intérêt direct de l'élucidation des représentations individuelles des élèves dans divers domaines essentiels de l'apprentissage de l'anglais. S'agissant notamment de la sensibilisation à la culture étrangère, il leur permettra de mieux saisir les effets des stéréotypes des élèves sur leur perception de la culture étrangère. En matière de développement des compétences de compréhension et d'expression, le recours à ce concept les conduira à s'appuyer sur les stratégies préalables des élèves comme nous l'avons souligné dans nos travaux de recherche précédents.

Néanmoins, il convient d'être vigilant quant aux écarts éventuels qui peuvent séparer les affirmations de principe des stagiaires au cours de cette phase d'élucidation et les actions qu'ils entreprennent lorsqu'ils ont la responsabilité d'une classe. Ainsi, l'importance qu'ils accordent à la découverte de la culture étrangère lors de cette étape préalable semble être démentie par la place tout à fait marginale qu'ils lui accordent lorsqu'ils sont mis à l'épreuve de la réalité. Un seul mémoire professionnel sur 130 est consacré à cette question au cours de ces trois dernières années. De plus, même si les stagiaires soulignent l'intérêt de développer les savoir-faire communicatifs des élèves, les dispositifs proposés dans leurs travaux de seconde année montrent que nombre d'entre eux continuent à privilégier l'acquisition des savoirs grammaticaux et lexicaux dans leurs stratégies d'enseignement.

\section{Conclusion}

Malgré ces réserves, l'exploration des représentations des stagiaires est constitutive du processus de formation. Elle est un moyen pour eux de devenir davantage acteur de leur propre formation. Elle constitue un outil permettant d'en orienter les contenus et les démarches en fonction des informations recueillies au cours de cette phase préliminaire. De surcroît, elle fait partie intégrante de ce processus dès lors qu'elle conduit les participants à construire le concept de "représentation». Même si cette prise de conscience n'exclut pas les résistances ultérieures que suscitera l'exposition à des données nouvelles, il convient de l'envisager comme une condition préalable indispensable à toute dynamique de changement et d'individualisation dans la formation des enseignants d'anglais. 


\section{BIBLIOGRAPHIE}

Astolfi, Jean-Pierre. 1993. « Comment les élèves apprennent-ils?». Sciences Humaines, 26-29.

Astolfi, Jean-Pierre. 1992. L'École pour apprendre. Paris : ESF.

Giordan, André et Gérard De Vecchi. 1987. Les Origines du savoir. Des conceptions des apprenants aux concepts scientifiques, Lausanne : Delachaux et Niestlé.

Haramboure, Françoise. 1994. «Formation initiale, formation continue en anglais. Projet de formation : Le cas des étudiants dans les filières technologiques de l'enseignement supérieur ». Thèse de doctorat préparée sous la direction de H. Fourtina, Université de Bordeaux 3.

Haramboure, Françoise. 1998. «Les Modules de pré-professionnalisation, effet de mode ?

Reconnaissance d'un besoin légitime? ». ASp, 19-22.

Williams, Marion and Robert Burden. 1997. Psychology for Language Teachers. A social constructivist approach. Cambridge : Cambridge University Press.

\section{ANNEXES}

Questionnaire

1. Certaines personnes ont un don pour l'enseignement.

2. Il faut que les enseignants stagiaires donnent des cours :

- dès le début de la formation initiale

- après une phase d'observation

3. Au cours de la formation initiale, les stagiaires doivent essentiellement assimiler les techniques qui ont fait leurs preuves dans les classes de langue étrangère.

4. La formation initiale suppose la mise en relation des théories de l'acquisition avec la pratique.

5. Je pense avoir des dispositions pour l'enseignement.

6. L'éclairage théorique fait partie intégrante de la formation initiale des enseignants.

7. La réussite de l'apprentissage dépend entièrement de la qualité de l'enseignement.

8. L'apprentissage dépend de l'activité intellectuelle des élèves.

9. Les erreurs doivent être corrigées

- immédiatement

- de façon sélective et différée.

10. L'erreur est une étape normale de l'apprentissage.

11. L'objectif essentiel de l'enseignement/apprentissage d'une langue étrangère est l'apprentissage de la grammaire et du vocabulaire. 
12. La connaissance de la culture cible est indispensable.

13. L'essentiel, c'est de mettre les élèves en situation de comprendre et de s'exprimer dans la langue étrangère le plus souvent possible.

14. L'enseignant doit garder l'initiative des échanges.

15. L'enseignement de la langue et celui de la culture étrangère ne peuvent être dissociés.

16. La communication en langue étrangère doit être évaluée en fonction de sa correction grammaticale

17. La prononciation doit être une préoccupation constante des enseignants.

18. L'essentiel est que les élèves s'expriment en LE.

19. L'utilisation de la L1 doit être exclue.

20. L'apprentissage dépend uniquement de la motivation des élèves.

\section{NOTES}

1. Cf Annexe

\section{RÉSUMÉS}

La prise de conscience par les enseignants stagiaires de leurs représentations sur le processus d'enseignement/apprentissage peut être considérée comme la première étape du processus de formation puisque ces dernières déterminent leur interprétation et appropriation des données nouvelles. Cette exploration contribue également à la construction du concept lui-même et constitue une source d'informations décisives pour les formateurs.

Raising awareness of teacher trainees' beliefs on the teaching/learning process can be considered as the preliminary stage of the training process since beliefs determine how participants interpret and appropriate new ideas from the course. This investigation also contributes to the very construction of the concept itself and represents a major source of information for teacher trainers.

\section{INDEX}

Keywords : awareness, belief, concept construction, teacher trainee, training process Mots-clés : construction du concept, enseignant stagiaire, prise de conscience, processus de formation, représentation 


\section{AUTEUR}

\section{FRANÇOISE HARAMBOURE}

Françoise Haramboure est maître de conférences à l'UFR des pays anglophones, université Michel de Montaigne-Bordeaux 3 où elle enseigne la didactique des langues étrangères. Elle est responsable de la préparation à l'épreuve sur dossier et membre du jury du CAPES externe d'anglais. f.haramboure@cegetel.net 